\title{
New uniqueness results for fractional differential equation with dependence on the first order derivative
}

\section{Zhenzhen Yue ${ }^{1}$ and Yumei Zou ${ }^{1 *}$ (i)}

\section{"Correspondence:}

sdzouym@126.com

1 Department of Statistics and

Finance, Shandong University of

Science and Technology, Qingdao, P.R. China

\begin{abstract}
In this paper, we study the uniqueness of solutions for a fractional differential equation with dependence on the first order derivative. By means of Banach's contraction mapping principle and a weighted norm in product space, sufficient conditions for the uniqueness of solutions are investigated. An example is given to illustrate the main results.
\end{abstract}

Keywords: Fractional differential equation; Uniqueness results

\section{Introduction}

In this paper, we consider the following Dirichlet boundary value problem for fractional differential equation:

$$
\left\{\begin{array}{l}
D_{0+}^{\alpha} u(t)+f\left(t, u(t), u^{\prime}(t)\right)=0, \quad t \in(0,1), \\
u(0)=u(1)=0
\end{array}\right.
$$

where $1<\alpha \leq 2$ and $f \in C\left([0,1] \times \mathbb{R}^{2}, \mathbb{R}\right)$. Here, $D_{0+}^{\alpha} u(t)$ denotes the standard RiemannLiouville fractional derivative of $u:[0,1] \rightarrow \mathbb{R}$ defined by

$$
D_{0^{+}}^{\alpha} u(t)=\frac{1}{\Gamma(n-\alpha)}\left(\frac{d}{d t}\right)^{n} \int_{0}^{t} \frac{u(s)}{(t-s)^{\alpha-n+1}} d s,
$$

where $n-1 \leq \alpha<n$, provided that the right-hand side is pointwise defined on $(0, \infty)$.

The problem of the existence of solutions for fractional differential equation with various boundary conditions has received an increased attention by using variational methods and critical point theory, the theory of coincidence degree, some well-known fixed point theorems, upper and lower solution method; see the monographs of Kilbas et al. [17], Miller and Ross [21], Podlubny [23], the papers [1-5, 12, 14-16, 18, 19, 24-27, 29-31, 33, $35,36,38,40-42]$, and the references therein. For example, Bai and Lü [5] considered the special case of BVP (1.1) that $f$ does not contain first order derivative term $u^{\prime}$ :

$$
\left\{\begin{array}{l}
D_{0+}^{\alpha} u(t)+f(t, u(t))=0, \quad t \in(0,1), \\
u(0)=u(1)=0,
\end{array}\right.
$$

\section{Springer}


where $1<\alpha \leq 2$ and $f \in C([0,1] \times[0,+\infty),[0,+\infty))$. The authors obtained the existence and multiplicity of positive solutions by means of the Krasnosel'skii fixed point theorem and the Leggett-Williams fixed point theorem. In [18], the existence of at least one solution for BVP (1.1) is proved by the Leray-Schauder continuation principle. In [2], the authors investigated the fractional differential equations

$$
\left\{\begin{array}{l}
D_{0+}^{\alpha} u(t)+f\left(t, u(t), D_{0+}^{\mu} u(t)\right)=0, \quad t \in(0,1), \\
u(0)=u(1)=0
\end{array}\right.
$$

where $1<\alpha<2, \mu>0$ are real numbers, $\alpha-\mu \geq 1, f$ is a Carathéodory function, and $f(t, x, y)$ is singular at $x=0$. The authors obtained the existence of positive solutions based on regularization and sequential techniques. Recently, the authors of [9] proved uniqueness results for BVP (1.2) by means of Banach's contraction mapping principle and the theory of linear operator.

At present, many papers are devoted to the uniqueness results for BVP; see [6-11, $13,20,22,28,34,37,39]$. Some nonlinear analytical techniques have been used to study the uniqueness of solutions for differential equation and differential systems such as the method of Banach's contraction mapping principle, fixed point theorems for mixed monotone operators, the maximal principle, $u_{0}$-positive operator, and linear operator theory. On the other hand, there are some papers studying fractional differential equations and fractional differential systems in which the fractional orders are involved in the nonlinearity, we refer the reader to $[2,18,32]$. Motivated by the results above, utilizing Banach's contraction mapping principle, we investigate the uniqueness result for solution of BVP (1.1).

It should noted here that our main result has various new system features. First of all, BVP (1.1) is reformulated as a fixed point problem for system of integral equations. Second, a weighted norm in product space is introduced. Third, the first order derivative is involved in the nonlinear terms.

Throughout the paper, we assume that the following condition holds:

(H) $f:[0,1] \times \mathbb{R}^{2} \rightarrow \mathbb{R}$ is a continuous function and there exist constants $A, B>0$ such that

$$
\left|f\left(t, u_{1}, v_{1}\right)-f\left(t, u_{2}, v_{2}\right)\right| \leq A\left|u_{1}-u_{2}\right|+B\left|v_{1}-v_{2}\right|, \quad t \in[0,1],
$$

for all $u_{1}, u_{2}, v_{1}, v_{2} \in \mathbb{R}$.

\section{Preliminaries}

Define two functions $G, G_{1}$ as follows:

$$
\begin{aligned}
& G(t, s)=\frac{1}{\Gamma(\alpha)} \begin{cases}(t(1-s))^{\alpha-1}-(t-s)^{\alpha-1}, & 0 \leq s \leq t \leq 1, \\
(t(1-s))^{\alpha-1}, & 0 \leq t \leq s \leq 1,\end{cases} \\
& G_{1}(t, s)=\frac{\alpha-1}{\Gamma(\alpha)} \begin{cases}t^{\alpha-2}(1-s)^{\alpha-1}-(t-s)^{\alpha-2}, & 0 \leq s \leq t \leq 1, \\
t^{\alpha-2}(1-s)^{\alpha-1}, & 0 \leq t \leq s \leq 1 .\end{cases}
\end{aligned}
$$


Lemma 2.1 ([5]) Let $G$ be as in (2.1). Then

$$
0 \leq G(t, s) \leq \frac{1}{\Gamma(\alpha)} t^{\alpha-1}(1-s)^{\alpha-1}, \quad t, s \in[0,1]
$$

After routine calculation we get the following four inequalities:

$$
\begin{aligned}
\Gamma(\alpha) \int_{0}^{1} G(t, s) s^{\alpha-1} d s \\
\quad=\int_{0}^{1}(t(1-s))^{\alpha-1} s^{\alpha-1} d s-\int_{0}^{t}(t-s)^{\alpha-1} s^{\alpha-1} d s \\
\quad=t^{\alpha-1} B(\alpha, \alpha)-t^{2 \alpha-1} \int_{0}^{1}(1-s)^{\alpha-1} s^{\alpha-1} d s \\
\quad=B(\alpha, \alpha) t^{\alpha-1}\left(1-t^{\alpha}\right) \leq B(\alpha, \alpha) t^{\alpha-1} \\
\Gamma(\alpha) \int_{0}^{1} G(t, s) s^{\alpha-2} d s \\
\quad=\int_{0}^{1}(t(1-s))^{\alpha-1} s^{\alpha-2} d s-\int_{0}^{t}(t-s)^{\alpha-1} s^{\alpha-2} d s \\
\quad=t^{\alpha-1} B(\alpha, \alpha-1)-t^{2 \alpha-2} B(\alpha, \alpha-1) \leq B(\alpha, \alpha-1) t^{\alpha-1} \\
\frac{\Gamma(\alpha)}{\alpha}-1 \int_{0}^{1}\left|G_{1}(t, s)\right| s^{\alpha-1} d s \\
\quad \leq t^{\alpha-2} \int_{0}^{1}(1-s)^{\alpha-1} s^{\alpha-1} d s+\int_{0}^{t}(t-s)^{\alpha-2} s^{\alpha-1} d s \\
\quad=t^{\alpha-2} B(\alpha, \alpha)+t^{2 \alpha-2} B(\alpha-1, \alpha) \\
\quad \leq(B(\alpha, \alpha)+B(\alpha-1, \alpha)) t^{\alpha-2},
\end{aligned}
$$

and

$$
\begin{aligned}
& \frac{\Gamma(\alpha)}{\alpha-1} \int_{0}^{1}\left|G_{1}(t, s)\right| s^{\alpha-2} d s \\
& \quad \leq t^{\alpha-2} \int_{0}^{1}(1-s)^{\alpha-1} s^{\alpha-2} d s+\int_{0}^{t}(t-s)^{\alpha-2} s^{\alpha-2} d s \\
& \quad=t^{\alpha-2} B(\alpha, \alpha-1)+t^{2 \alpha-3} B(\alpha-1, \alpha-1) \\
& \quad \leq(B(\alpha, \alpha-1)+B(\alpha-1, \alpha-1)) t^{\alpha-2} .
\end{aligned}
$$

Here, $B(\alpha, \beta)$ is the beta function defined by the Euler integral:

$$
B(\alpha, \beta)=\int_{0}^{1} s^{\alpha-1}(1-s)^{\beta-1} d s .
$$

Lemma $2.2([5,18])$ Let $h \in C[0,1]$. Then

$$
u(t)=\int_{0}^{1} G(t, s) h(s) d s
$$


is the unique solution of

$$
\left\{\begin{array}{l}
D_{0+}^{\alpha} u(t)+h(t)=0, \quad t \in(0,1) \\
u(0)=u(1)=0 .
\end{array}\right.
$$

Moreover, $u^{\prime} \in C(0,1] \cap A C_{\mathrm{loc}}(0,1], \lim _{t \rightarrow 0^{+}} t^{2-\alpha} u^{\prime}(t)$ exists and satisfies

$$
u^{\prime}(t)=\int_{0}^{1} G_{1}(t, s) h(s) d s
$$

We set $E_{1}=C[0,1]$ with the usual maximum norm denoted by $\|u\|_{E_{1}}=\max _{t \in(0,1]}|u(t)|$. Consider the Banach space

$$
E_{2}=\left\{v \in C(0,1]: \lim _{t \rightarrow 0^{+}} t^{2-\alpha} v(t) \text { exists }\right\}
$$

with the norm $\|v\|_{E_{2}}=\sup _{t \in(0,1]}\left|t^{2-\alpha} v(t)\right|$. Then $E_{1} \times E_{2}$ is a Banach space with the norm

$$
\|(u, v)\|_{E_{1} \times E_{2}}=\max \left\{\|u\|_{E_{1}},\|v\|_{E_{2}}\right\} .
$$

According to Lemma 2.2, BVP (1.1) has a solution $u=u(t)$ if and only if $(u, v) \in E_{1} \times E_{2}$ solves the following integral equations:

$$
\left\{\begin{array}{l}
u(t)=\int_{0}^{1} G(t, s) f(s, u(s), v(s)) d s, \\
v(t)=\int_{0}^{1} G_{1}(t, s) f(s, u(s), v(s)) d s,
\end{array}\right.
$$

with $v=u^{\prime}$. Define an operator $T$ by

$$
T(u, v)=\left(T_{1}(u, v), T_{2}(u, v)\right), \quad(u, v) \in E_{1} \times E_{2},
$$

where operators $T_{1}, T_{2}$ are defined by

$$
\begin{aligned}
& T_{1}(u, v)(t)=\int_{0}^{1} G(t, s) f(s, u(s), v(s)) d s, \quad(u, v) \in E_{1} \times E_{2}, \\
& T_{2}(u, v)(t)=\int_{0}^{1} G_{1}(t, s) f(s, u(s), v(s)) d s, \quad(u, v) \in E_{1} \times E_{2},
\end{aligned}
$$

respectively. For $(u, v) \in E$, by Lemma 2.1 and $(H)$, we have

$$
\begin{aligned}
& \left|T_{1}(u, v)(t)\right| \\
& \quad \leq \int_{0}^{1} G(t, s)|f(s, u(s), v(s))-f(s, 0,0)| d s+\int_{0}^{1} G(t, s)|f(s, 0,0)| d s \\
& \quad \leq \frac{t^{\alpha-1}}{\Gamma(\alpha)} \int_{0}^{1}(A|u(s)|+B|v(s)|)(1-s)^{\alpha-1} d s+\frac{t^{\alpha-1}}{\Gamma(\alpha)} \int_{0}^{1}|f(s, 0,0)| d s \\
& \quad \leq \frac{A\|u\|_{E_{1}}}{\Gamma(\alpha+1)} t^{\alpha-1}+\frac{B\|v\|_{E_{2}}}{\Gamma(\alpha)} \int_{0}^{1} s^{\alpha-2}(1-s)^{\alpha-1} d s \cdot t^{\alpha-1}
\end{aligned}
$$




$$
\begin{aligned}
& +\frac{t^{\alpha-1}}{\Gamma(\alpha)} \int_{0}^{1}(1-s)^{\alpha-1}|f(s, 0,0)| d s \\
= & \left(\frac{A\|u\|_{E_{1}}}{\Gamma(\alpha+1)}+\frac{B \Gamma(\alpha-1)\|v\|_{E_{2}}}{\Gamma(2 \alpha-1)}+\frac{1}{\Gamma(\alpha)} \int_{0}^{1}|f(s, 0,0)| d s\right) t^{\alpha-1},
\end{aligned}
$$

which implies that $T_{1}$ is well defined on $E$ and $T_{1}(u, v) \in E_{1}$. In the same way, we can prove that $T_{2}$ is well defined on $E$ and $T_{2}(u, v) \in E_{2}$ for all $(u, v) \in E$. Thus, the existence of a solution of BVP (1.1) is equivalent to the existence of a fixed point of $T$ on $E_{1} \times E_{2}$.

It follows from (2.7) that $T_{1}$ maps all of $E_{1} \times E_{2}$ into the following vector subspace of $E_{1}$ :

$$
E_{3}=\left\{u \in E_{1}: \frac{|u(t)|}{t^{\alpha-1}} \text { are bounded for } t \in[0,1]\right\} .
$$

Clearly, $E_{3}$ is a Banach space with the norm

$$
\|u\|_{E_{3}}=\sup _{t \in(0,1]} t^{1-\alpha}|u(t)| .
$$

Hence, in the following we only need to consider the fixed points of $T$ in the Banach space $E=E_{3} \times E_{2}$ with the weighted norm

$$
\|(u, v)\|_{E}=\max \left\{\|u\|_{E_{3}}, \frac{\|v\|_{E_{2}}}{\theta}\right\}
$$

with a constant $\theta>0$.

Lemma 2.3 Let $a, d \in[0,1), b, c \in[0,+\infty)$ with $(1-d)(1-a)>b c$. Then the system of inequalities

$$
\left\{\begin{array}{l}
a+b \theta \leq \lambda, \\
\frac{c}{\theta}+d \leq \lambda
\end{array}\right.
$$

has a solution $(\lambda, \theta)$ with $\lambda \in(0,1)$ and $\theta>0$.

Proof For the case $b c=0$, we may take $\lambda=\max \left\{\frac{d+1}{2}, \frac{d+1}{2}\right\}$. So it remains to consider the case $b c \neq 0$. Let

$$
\phi(x)=(x-d)(x-a)-b c, \quad x \in \mathbb{R} .
$$

It follows from the derivative of $\phi(x)$ that $\phi(x)$ is increasing on $\left[\frac{a+d}{2}, 1\right]$. With the help of the locally sign-preserving property of $\phi(x)$, we conclude that there exists $\lambda \in\left[\frac{a+d}{2}, 1\right)$ such that

$$
(\lambda-d)(\lambda-a) \geq b c
$$

The above inequality is equivalent to $\frac{c}{\lambda-d} \leq \frac{\lambda-a}{b}$. Therefore (2.8) holds for $\theta \in\left[\frac{c}{\lambda-d}, \frac{\lambda-a}{b}\right]$. 


\section{Main results}

Set

$$
\begin{aligned}
& a_{11}=\frac{A \Gamma(\alpha)}{\Gamma(2 \alpha)}, \quad a_{21}=\frac{A(\alpha-1)(B(\alpha, \alpha)+B(\alpha-1, \alpha))}{\Gamma(\alpha)}, \\
& a_{12}=\frac{B \Gamma(\alpha-1)}{\Gamma(2 \alpha-1)}, \quad a_{22}=\frac{B(\alpha-1)(B(\alpha, \alpha-1)+B(\alpha-1, \alpha-1))}{\Gamma(\alpha)} .
\end{aligned}
$$

Now, we show that a uniqueness result follows from Banach's contraction mapping principle.

Theorem 3.1 Suppose that condition (H) holds. In addition, we assume that the four constants $a_{11}, a_{12}, a_{21}, a_{22}$ satisfy

$$
a_{11}<1, \quad a_{22}<1,
$$

and

$$
\left(1-a_{11}\right)\left(1-a_{22}\right)>a_{12} a_{21}
$$

Then BVP (1.1) has a unique solution.

Proof It follows from Lemma 2.3 that there exist $\lambda \in(0,1)$ and $\theta>0$ such that

$$
\left\{\begin{array}{l}
a_{11}+a_{12} \theta \leq \lambda, \\
\frac{a_{21}}{\theta}+a_{22} \leq \lambda
\end{array}\right.
$$

We shall apply Banach's contraction mapping principle in $E=E_{3} \times E_{2}$ endowed with the weighted norm

$$
\|(u, v)\|_{E}=\max \left\{\|u\|_{E_{3}}, \frac{\|v\|_{E_{2}}}{\theta}\right\}
$$

with $\theta>0$ as in (3.1). More precisely, we prove that

$$
\left\|T\left(u_{1}, v_{1}\right)-T\left(u_{2}, v_{2}\right)\right\|_{E} \leq \lambda\left\|\left(u_{1}, v_{1}\right)-\left(u_{2}, v_{2}\right)\right\|_{E}
$$

for all $\left(u_{1}, v_{1}\right),\left(u_{2}, v_{2}\right) \in E$. In fact, by (2.3), (2.4), and (3.1), we have

$$
\begin{aligned}
& \left|T_{1}\left(u_{1}, v_{1}\right)(t)-T_{1}\left(u_{2}, v_{2}\right)(t)\right| \\
& \quad \leq \int_{0}^{1} G(t, s)\left|f\left(s, u_{1}(s), v_{1}(s)\right)-f\left(s, u_{2}(s), v_{2}(s)\right)\right| d s \\
& \quad \leq \int_{0}^{1} G(t, s)\left(A\left|u_{1}(s)-u_{2}(s)\right|+B\left|v_{1}(s)-v_{2}(s)\right|\right) d s \\
& \quad \leq \int_{0}^{1} G(t, s)\left(A\left\|u_{1}-u_{2}\right\|_{E_{3}} s^{\alpha-1}+B\left\|v_{1}-v_{2}\right\|_{E_{2}} s^{\alpha-2}\right) d s
\end{aligned}
$$




$$
\begin{aligned}
& \leq\left(a_{11}\left\|u_{1}-u_{2}\right\|_{E_{3}}+a_{12} \theta \frac{\left\|v_{1}-v_{2}\right\|_{E_{2}}}{\theta}\right) \cdot t^{\alpha-1} \\
& \leq \lambda\left\|\left(u_{1}, v_{1}\right)-\left(u_{2}, v_{2}\right)\right\|_{E} \cdot t^{\alpha-1} .
\end{aligned}
$$

Hence, from the definition of $\|\cdot\|_{E_{3}}$, we have

$$
\left\|T_{1}\left(u_{1}, v_{1}\right)-T_{1}\left(u_{2}, v_{2}\right)\right\|_{E_{3}} \leq \lambda\left\|\left(u_{1}, v_{1}\right)-\left(u_{2}, v_{2}\right)\right\|_{E} .
$$

Similar, we have

$$
\begin{aligned}
\frac{1}{\theta} \mid & T_{2}\left(u_{1}, v_{1}\right)(t)-T_{2}\left(u_{2}, v_{2}\right)(t) \mid \\
& \leq \frac{1}{\theta} \int_{0}^{1}\left|G_{1}(t, s)\right|\left|f\left(s, u_{1}(s), v_{1}(s)\right)-f\left(s, u_{2}(s), v_{2}(s)\right)\right| d s \\
& \leq \frac{1}{\theta} \int_{0}^{1}\left|G_{1}(t, s)\right|\left(A\left|u_{1}(s)-u_{2}(s)\right|+B\left|v_{1}(s)-v_{2}(s)\right|\right) d s \\
& \leq \frac{1}{\theta} \int_{0}^{1}\left|G_{1}(t, s)\right|\left(A\left\|u_{1}-u_{2}\right\|_{E_{3}} s^{\alpha-1}+B\left\|v_{1}-v_{2}\right\|_{E_{2}} s^{\alpha-2}\right) d s \\
& \leq\left(\frac{1}{\theta} \cdot a_{21}\left\|u_{1}-u_{2}\right\|_{E_{3}}+a_{22} \frac{\left\|v_{1}-v_{2}\right\|_{E_{2}}}{\theta}\right) \cdot t^{\alpha-2} \\
& \leq \lambda\left\|\left(u_{1}, v_{1}\right)-\left(u_{2}, v_{2}\right)\right\|_{E} \cdot t^{\alpha-2} .
\end{aligned}
$$

Thus, we have

$$
\frac{1}{\theta}\left\|T_{2}\left(u_{1}, v_{1}\right)-T_{2}\left(u_{2}, v_{2}\right)\right\|_{E_{2}} \leq \lambda\left\|\left(u_{1}, v_{1}\right)-\left(u_{2}, v_{2}\right)\right\|_{E} .
$$

Now, both inequalities (3.2) and (3.3) can be rewritten equivalently as

$$
\left\|T\left(u_{1}, v_{1}\right)-T\left(u_{2}, v_{2}\right)\right\|_{E} \leq \lambda\left\|\left(u_{1}, v_{1}\right)-\left(u_{2}, v_{2}\right)\right\|_{E} .
$$

Note that $\lambda \in(0,1)$. The uniqueness result follows from Banach's contraction mapping principle.

In what follows, we give an example to illustrate the application of our results.

Example 3.1 Consider the BVP

$$
\left\{\begin{array}{l}
D_{0+}^{\frac{3}{2}} u(t)+\frac{1}{3 \sqrt{\pi}} \sin \left(6 u(t)+u^{\prime}(t)\right)+h(t)=0, \\
u(0)=u(1)=0,
\end{array}\right.
$$

where $t \in[0,1], \alpha=\frac{3}{2}$, and $h \in C[0,1]$. Let $f(t, u, v)=\frac{1}{3 \sqrt{\pi}} \sin (6 u+v)+h(t)$. It is easy to see that $\left|f\left(t, u_{1}, v_{1}\right)-f\left(t, u_{2}, v_{2}\right)\right| \leq \frac{2}{\sqrt{\pi}}\left|u_{1}-u_{2}\right|+\frac{1}{3 \sqrt{\pi}}\left|v_{1}-v_{2}\right|$ for all $t \in[0,1], u_{1}, u_{2}, v_{1}, v_{2} \in \mathbb{R}$. Then, for $\alpha=\frac{3}{2}, A=\frac{2}{\sqrt{\pi}}$, and $B=\frac{1}{3 \sqrt{\pi}}$, we have that $a_{11}=\frac{1}{2}, a_{12}=\frac{1}{6}, a_{21}=\frac{5}{4}$, and $a_{22}=\frac{1}{2}$. So $\left(1-a_{11}\right)\left(1-a_{22}\right)>a_{12} a_{21}$ holds, and from Theorem 3.1 BVP (3.4) has a unique solution. 


\section{Acknowledgements}

The authors sincerely thank the editors and anonymous referees for the careful reading of the original manuscript and for valuable comments, which have improved the quality of our work.

\section{Funding}

This work was partially supported by the Natural Science Foundation of China (11801322), the Shandong Natural Science Foundation (ZR2018MA011).

\section{Competing interests}

The authors declare that they have no competing interests.

\section{Authors' contributions}

The authors have made the same contribution. All authors read and approved the final manuscript.

\section{Publisher's Note}

Springer Nature remains neutral with regard to jurisdictional claims in published maps and institutional affiliations.

Received: 4 September 2018 Accepted: 6 December 2018 Published online: 29 January 2019

\section{References}

1. Agarwal, R.P., Benchohra, M., Hamani, S.: A Survey on Existence Results for Boundary Value Problems of Nonlinear Fractional Differential Equations and Inclusions. Acta Appl. Math. 109, 973-1033 (2010)

2. Agarwal, R.P., O'Regan, D., Staněk, S.: Positive solutions for Dirichlet problems of singular nonlinear fractional differential equations. J. Math. Anal. Appl. 371, 57-68 (2010)

3. Ahmad, B., Alsaedi, A.: Existence and uniqueness of solutions for coupled systems of higher-order nonlinear fractional differential equations. Fixed Point Theory Appl. 2010, 364560 (2010)

4. Bai, Z.: On positive solutions of a nonlocal fractional boundary value problem. Nonlinear Anal. 72, 916-924 (2010)

5. Bai, Z., Lü, H.: Positive solutions for boundary value problems of nonlinear fractional differential equation. J. Math. Anal. Appl. 311, 495-505 (2005)

6. Bai, Z., Sun, S., Chen, Y.: The Existence and Uniqueness of a Class of Fractional Differential Equations. Abstr. Appl. Anal. 2014, $486040(2014)$

7. Cui, Y.: Uniqueness of solution for boundary value problems for fractional differential equations. Appl. Math. Lett. 51 48-54 (2016)

8. Cui, Y., Liu, L., Zhang, X.: Uniqueness and existence of positive solutions for singular differential systems with coupled integral boundary value problems. Abstr. Appl. Anal. 2013, 340487 (2013)

9. Cui, Y., Ma, W., Sun, Q., Su, X.: New uniqueness results for boundary value problem of fractional differential equation. Nonlinear Anal., Model. Control 23(1), 31-39 (2018). https://doi.org/10.15388/NA.2018.1.3

10. Cui, Y., Ma, W., Wang, X., Su, X.: Uniqueness theorem of differential system with coupled integral boundary conditions Electron. J. Qual. Theory Differ. Equ. 2018(9), 1 (2018). https://doi.org/10.14232/ejqtde.2018.1.9

11. Cui, Y., Zou, Y.: An existence and uniqueness theorem for a second order nonlinear system with coupled integral boundary value conditions. Appl. Math. Comput. 256, 438-444 (2015)

12. Dong, X., Bai, Z., Zhang, S.: Positive solutions to boundary value problems of $\mathrm{p}$-Laplacian with fractional derivative. Bound. Value Probl. 2017, 5 (2017)

13. Graef, J., Kong, L., Kong, Q., Wang, M.: Uniqueness of positive solutions of fractional boundary value problems with nonhomogeneous integral boundary conditions. Fract. Calc. Appl. Anal. 15(3), 509-528 (2012)

14. Guo, L., Liu, L., Wu, Y.: Existence of positive solutions for singular fractional differential equations with infinite-point boundary conditions. Nonlinear Anal., Model. Control 21(5), 635-650 (2016)

15. Hao, X., Sun, H., Liu, L.: Existence results for fractional integral boundary value problem involving fractional derivatives on an infinite interval. Math. Methods Appl. Sci. 41(16), 6984-6996 (2018). https://doi.org/10.1002/mma.5210

16. Hao, X., Zuo, M., Liu, L.: Multiple positive solutions for a system of impulsive integral boundary value problems with sign-changing nonlinearities. Appl. Math. Lett. 82, 24-31 (2018)

17. Kilbas, A.A., Srivastava, H.M., Trujillo, J.J.: Theory and Applications of Fractional Differential Equations. North-Holland Mathematics Studies, vol. 204. Elsevier, Amsterdam (2006)

18. Kosmatov, N.: A singular boundary value problem for nonlinear differential equations of fractional order. J. Appl. Math. Comput. 29, 125-135 (2009)

19. Liu, L., Zhang, X., Jiang, J., Wu, Y.: The unique solution of a class of sum mixed monotone operator equations and its application to fractional boundary value problems. J. Nonlinear Sci. Appl. 9(5), 2943-2958 (2016)

20. Meng, S., Cui, Y.: The Uniqueness Theorem of the Solution for a Class of Differential Systems with Coupled Integral Boundary Conditions. Discrete Dyn. Nat. Soc. 2018, Article ID 9601868 (2018)

21. Miller, K.S., Ross, B.: An Introduction to the Fractional Calculus and Fractional Differential Equation. Wiley, New York (1993)

22. Min, D., Liu, L., Wu, Y.: Uniqueness of positive solutions for the singular fractional differential equations involving integral boundary value conditions. Bound. Value Probl. 2018, Article ID 23 (2018)

23. Podlubny, I.: Fractional Differential Equations, Mathematics in Science and Engineering. Academic Press, New York (1999)

24. Pu, R., Zhang, X., Cui, Y., Li, P., Wang, W.: Positive solutions for singular semipositone fractional differential equation subject to multipoint boundary conditions. J. Funct. Spaces 2017, Article ID 5892616 (2017)

25. Qi, T., Liu, Y., Cui, Y.: Existence of solutions for a class of coupled fractional differential systems with nonlocal boundary conditions. J. Funct. Spaces 2017, 6703860 (2017)

26. Qi, T., Liu, Y., Zou, Y.: Existence result for a class of coupled fractional differential systems with integral boundary value conditions. J. Nonlinear Sci. Appl. 10, 4034-4045 (2017) 
27. Sun, Q., Ji, H., Cui, Y.: Positive solutions for boundary value problems of fractional differential equation with integral boundary conditions. J. Funct. Spaces 2018, Article ID 6461930 (2018)

28. Ur Rehman, M., Ali Khan, R.: Existence and uniqueness of solutions for multi-point boundary value problems for fractional differential equations. Appl. Math. Lett. 23, 1038-1044 (2010)

29. Wang, Y., Liu, L.: Uniqueness and existence of positive solutions for the fractional integro-differential equation. Bound. Value Probl. 2017, 12 (2017)

30. Wu, J., Zhang, X., Liu, L., Wu, Y., Cui, Y.: The convergence analysis and error estimation for unique solution of a p-Laplacian fractional differential equation with singular decreasing nonlinearity. Bound. Value Probl. 2018, 82 (2018)

31. Xu, M., Han, Z:: Positive solutions for integral boundary value problem of two-term fractional differential equations. Bound. Value Probl. 2018, 100 (2018)

32. Zhang, $X .$, Liu, L., Wu, Y.: The uniqueness of positive solution for a singular fractional differential system involving derivatives. Commun. Nonlinear Sci. Numer. Simul. 18, 1400-1409 (2013)

33. Zhang, X., Liu, L., Wu, Y., Cui, Y.: Entire blow-up solutions for a quasilinear $p$-Laplacian Schrodinger equation with a non-square diffusion term. Appl. Math. Lett. 74, 85-93 (2017)

34. Zhang, X., Liu, L., Wu, Y., Zou, Y.: Existence and uniqueness of solutions for systems of fractional differential equations with Riemann-Stieltjes integral boundary condition. Adv. Differ. Equ. 2018, Article ID 204 (2018) https://doi.org/10.1186/s13662-018-1650-7

35. Zhang, X., Shao, Z., Zhong, Q.: Positive solutions for semipositone $(k, n-k)$ conjugate boundary value problems with singularities on space variables. Appl. Math. Lett. 72, 50-57 (2017)

36. Zhang, X., Wu, Y., Cui, Y.: Existence and nonexistence of blow-up solutions for a Schrodinger equation involving a nonlinear operator. Appl. Math. Lett. 82, 85-91 (2018)

37. Zhang, X., Zhong, Q.: Uniqueness of solution for higher-order fractional differential equations with conjugate type integral conditions. Fract. Calc. Appl. Anal. 20(6), 1471-1484 (2017)

38. Zhang, X., Zhong, Q: Triple positive solutions for nonlocal fractional differential equations with singularities both on time and space variables. Appl. Math. Lett. 80, 12-19 (2018)

39. Zou, Y., He, G.: On the uniqueness of solutions for a class of fractional differential equations. Appl. Math. Lett. 74 68-73 (2017)

40. Zou, Y., He, G.: A fixed point theorem for systems of nonlinear operator equations and applications to $\left(p_{1}, p_{2}\right)$-Laplacian system. Mediterr. J. Math. 15, 74 (2018)

41. Zou, Y., Liu, L., Cui, Y.: The existence of solutions for four-point coupled boundary value problems of fractional differential equations at resonance. Abstr. Appl. Anal. 2014, 314083 (2014)

42. Zuo, M., Hao, X., Liu, L., Cui, Y.: Existence results for impulsive fractional integro-differential equation of mixed type with constant coefficient and antiperiodic boundary conditions. Bound. Value Probl. 2017, 161 (2017)

\section{Submit your manuscript to a SpringerOpen ${ }^{\circ}$ journal and benefit from:}

- Convenient online submission

- Rigorous peer review

- Open access: articles freely available online

- High visibility within the field

- Retaining the copyright to your article

Submit your next manuscript at $\gg$ springeropen.com 